\title{
Primary and Backup Paths Optimal Design for Traffic Engineering in Hybrid IGP/MPLS Networks
}

\author{
Anna Mereu*, Davide Cherubini $^{\dagger}$, Alessandra Fanni*, Antonio Frangioni ${ }^{\ddagger}$ \\ *Department of Electrical and Electronic Engineering \\ University of Cagliari, Piazza d'Armi, 09123 Cagliari, Italy \\ Email: anna.mereu,fanni@diee.unica.it \\ ${ }^{\dagger}$ Tiscali International Network R \& D \\ Loc. "Sa Illetta", 09121 Cagliari, Italy \\ Email: davide.cherubini@alcatel-lucent.com \\ $\ddagger$ Department of Computer Science \\ University of Pisa, Largo B. Pontecorvo 3, I-56127 Pisa, Italy \\ Email: frangio@di.unipi.it
}

\begin{abstract}
The paper describes an optimization model which aims at minimizing the maximum link utilization of IP telecommunication networks under the joint use of the traditional IGP protocols and the more sophisticated MPLS-TE technology. The survivability of the network is taken into account in the optimization process implementing the path restoration scheme. This scheme benefits of the Fast Re-Route (FRR) capability allowing service providers to offer high availability and high revenue SLAs (Service Level Agreements). The hybrid IGP/MPLS approach relies on the formulation of an innovative Linear Programming mathematical model that, while optimizing the network utilization, provides optimal user performance, efficient use of network resources, and $100 \%$ survivability in case of single link failure. The possibility of performing an optimal exploitation of the network resources throughout the joint use of the IGP and MPLS protocols provides a flexible tool for the ISP (Internet Service Provider) networks traffic engineers.

The efficiency of the proposed approach is validated by a wide experimentation performed on synthetic and real networks. The obtained results show that a limited number of LSP tunnels have to be set up in order to significantly reduce the congestion level of the network while at the same time guaranteeing the survivability of the network.
\end{abstract}

Index Terms-Network Reliability, Optimal Design, Path Restoration, Routing

\section{INTRODUCTION}

The exponential growth of the Internet that has taken place in the last years and the subsequent birth of Quality of Service (QoS) requirements have led the Network Engineers to build very large scale networks at their best, trying to understand beforehand and react just in time to loss of performance and possible failure events, which can be technically and economically harmful. One could potentially think about solving the well known General Routing Problem (GRP) [1], whose aim is to perform the routing of the traffic throughout the network by optimizing the use of the available network resources without taking into account the protocols restrictions. With the IGP protocol the implementation of the solution of the GRP is

D. Cherubini is now with Alcatel-Lucent Bell Labs Ireland \& UK, Blanchardstown Industrial Park, Blanchardstown, Ireland unpracticable: the difficulty relies on the fact that, as known, the IGP protocols do not support a free distribution of flows because the routing of the demands is completely determined by the weights of the links, that have to be integers and can vary in the range from 1 to $2^{24}$. In [2], the problem of finding the optimal set of weights for the IS-IS/OSPF protocol is shown to be NP-hard and the optimal value cannot be approximated with a factor better than $3 / 2$.

On the contrary, Multi-Protocol Label Switching (MPLSTE) networks enable ISPs to adopt QoS policies, setting up constrained label switched paths (LSPs). Furthermore, network survivability techniques have been developed to guarantee seamless communication services in case of network failures. Traffic management and restoration strategies are usually adopted in order to make a backbone network survivable, i.e., the network traffic load has to be distributed in such a way that a failure has the minimum (eventually, null) impact when it occurs; moreover, the traffic demands affected by the failure have to be suitably restored. In an MPLS network one could specify explicitly one or several paths for each traffic demand from a source to a destination starting from the optimal solution of the GRP. However, the use of an MPLS full mesh approach is not recommended. The main drawback of this approach is related to the scalability, which allows to use it only for small networks. In fact, for a network composed by $n$ nodes, $n \times(n-1)$ LSPs should be established in order to route the traffic between every pair of nodes. For these reasons the research interest has been focussed towards the search of an optimal set of complementary LSP tunnels to be used in combination with the IGP protocol.

The TE routing problem that we address in this work performs an hybrid IGP/MPLS routing optimization by guaranteeing the survivability of the network. Given the topology of the network to be engineered, and the traffic matrix to be routed on it, the problem is to find a routing scheme that optimizes the network usage, with the following joint goals: an efficient use of the network resources and a $100 \%$ level of survivability in case of single link failure. This means that, 
starting from a certain traffic matrix, the output of our model is a set of optimal primary LSP tunnels corresponding to a set of demands that will be routed with the MPLS protocol, while the others will be routed along the shortest paths computed with the IGP weights. The setting of the optimal explicit MPLS paths together with the implicit primary paths (IGP shortest paths) guarantees a $100 \%$ level of survivability.

In order to avoid congestion scenarios due to link failures, two different restoration schemes may be implemented. With the link restoration scheme, the traffic is routed through a back-up path that is found to protect the failed link; on the other hand, with the path restoration scheme, if a failure is present along a path, the traffic is routed in a completely new path that starts from the origin node of the commodity and terminates in the destination node of the commodity. In [3] the authors presented a linear programming model performing an hybrid IGP/MPLS link restoration scheme. The model has been built according to the well known multi commodity min cost flow problem implemented with a node-arc formulation. Results have shown that the congestion levels of the network decrease substantially by setting up the set of optimal LSP with respect to the IGP routing protocol alone. However, a link restoration procedure may not always result in an efficient use of the network. In fact, the link restoration scheme may suffer of backhauling, that is a situation in which some links can be used more than once in a path. Moreover, the link restoration scheme results in a more complex recovery procedure for the network manager if compared to that required by the path restoration scheme: further details on the practical implementation of the restoration scheme will be given later on. For these reasons, in this work we implement the hybrid IGP/MPLS path restoration procedure.

The remainder of this paper is organized as follows. Section II presents a technological overview of the problem. Related works are presented in Section III. In Section IV we describe the proposed optimization model, in Section $\mathrm{V}$ we present the results and we conclude in Section VI.

\section{TeChNOLOGy OVERVIEW}

\section{A. Protocols}

IP backbone networks make use of IGP routing protocols, such as IS-IS and OSPF, in order to compute the routes from each origin node to each destination node of the traffic demands by associating a suitable metric value with each link of the network. Once the full topology of the network is known, the routers apply the Dijkstra algorithm [4] in order to compute the shortest path linking each origin-destination pair. If the network topology changes, then the protocol recalculates the shortest routes using the Dijkstra algorithm. When Equivalent Cost Multiple Paths (ECMP) are enabled, the traffic flows will be equally split among the shortest paths.

The introduction of the Multi-Protocol Label Switching Traffic Engineering (MPLS-TE) technology has improved the dataflow management due to the traditional routing protocols. IP routing protocols, in fact, compute routing paths assigning a single metric per link and using destination-based routing not providing a general and scalable method for explicitly routing traffic. On the contrary, MPLS/MPLS-TE networks can support destination-based and explicit routing simultaneously. These two different kinds of LSPs can be named "Implicit LSP" and "Explicit LSP", respectively. MPLS-TE networks are also able to minimize network congestion, so improving the overall network performance, reducing the impact of network failures and increasing service availability [5]. MPLSTE provides mechanisms to quickly find an alternate path if the primary path is no longer available (typically due to a node or link failure): the so-called Fast Re-Route (FRR) capability [6].

\section{B. Faults and Survivability}

The backbone networks are usually well-engineered and properly provisioned, leading to very low packet losses and negligible queuing delays. However, failures occur almost every day [7]. An understanding of their characteristics, properties and effects is extremely valuable. Accordingly to [8], failures are classified into two main groups: those resulting from scheduled maintenance activities $(20 \%)$ and those generated by unplanned failures $(80 \%)$. This latter set is mainly composed of single link failures $(70 \%)$. This is the type of failures we will address in our modeling proposal.

In literature, several restoration schemes have been presented: link restoration and path restoration schemes are the most important two. With the link restoration scheme the traffic that was carried by the link that has failed is rerouted from the source node to target node of that link. In practice, this means that the original route for the traffic that was carried by the failed link has to be modified by the replacement of the failed link with an alternate path that connects the two endpoints of the link [9]. On the other hand, path restoration is performed throughout a rerouting of the traffic back from the source node to the destination node of the demand through a possibly (but not required) totally disjoint path.

Both the restoration schemes can be practically implemented in the MPLS-TE technology throughout Fast ReRouting procedures. In Fast Re-Routing primary paths are set for each traffic demand along with a set of backup paths that are pre-computed but not pre-allocated: if the failure of a link affects a primary path, the traffic is switched to the backup path. When setting up a primary tunnel LSP for a certain traffic demand, the network manager can also define a dynamic protection procedure for it so that, whenever the failure of a link occurs, the traffic that was flowing in the primary path is sent to the backup path that had been computed in advance according to the shortest path computed with the weights of the IGP protocol. This procedure actually performs the path restoration technique. On the other hand, in order to implement the link restoration scheme with the Fast Re-Routing strategy, the primary LSP tunnel has to be built as the composition of single link LSPs whose protection mechanism has been defined dynamic, as explained above. In this way, the failure of a link is managed by substituting the failed link with a backup path from the link origin to the link destination node rather 
than from the ingress to the egress nodes of the commodity affected by the fault.

\section{RELATED WORK}

In literature, many papers have been published that address the problem of optimizing traffic routing using IS-IS/OSPF protocols. In particular, in [10] the authors adopted the General Routing Problem (GRP) [1], where the minimum cost multi commodity flow problem uses as objective function the sum of piecewise linear cost functions associated to the links. In the last few years, Tabu Search [11] [12], Genetic algorithms [13] [14], and tailored heuristics [15] [16], have been proposed to solve the same problem. The results of these papers show that IS-IS/OSPF routing does not perform as well as optimal MPLS-TE routing, even if optimal metrics are set.

Many papers also appeared, which focus on the optimization of routing of type MPLS. The main objective of these papers is to find an "optimal" LSPs mesh deployment according to different objective functions. In particular, in [17], a Tabu Search algorithm is proposed to find a layout of MPLS paths with the minimum number of hops, whereas in [18] the MPLS routing problem is modeled in terms of an offline multiobjective Mixed Integer Linear Programming model, that looks for the best trade-off between the minimal routing delay, the optimal load balancing, and the minimal splitting of traffic trunks. In [19] and in [20] a multipath adaptive traffic engineering mechanism is introduced. The approach aims at avoiding the network congestion by adaptively balancing the load among multiple paths based on measurements and analysis of the path level.

To the best of our knowledge, there are only a few publications that deal with hybrid IGP/MPLS scenarios for traffic engineering. Most of these papers present a two phase (hierarchical) approach by optimizing separately the two technologies. In particular, in [21] the author proposes a twophases algorithm: the IGP optimization is firstly performed using Simulated Annealing (SA) heuristics, then a set of complementary LSP tunnels are computed to improve the previous IGP solution using a Mixed Integer Programming model. The results show that the use of LSPs greatly improve the network level. In [22] the authors propose a genetic algorithm to find the optimal set of LSP paths to be used in combination with the IGP protocol: the idea is to rely on the IP native routing as much as possible and use MPLS only if necessary. In [23] a Simulated Annealing meta-heuristic is used to select a small number of LSPs under different objective functions. The idea is to perform a search for an optimal set of LSP tunnels starting from an initial candidate path list that is built according to specific requirements, such as the maximum number of hops.

All these works demonstrate that a small number of LSPs is always sufficient to obtain a better exploitation of the network resources both in terms of decreasing the congestion level and balancing the traffic load in the network.

It is worth noting that the aforementioned papers do not take into consideration the survivability issue embedded in the overall optimization process. In the majority of the literature, survivability is addressed by simulation and/or heuristic approach (some examples can be found in [24] as far as the IS-IS technology is concerned whereas [25] and [26] refer to the MPLS protocol).

In [3] the authors address the hybrid IGP/MPLS optimization problem taking into account the survivability condition by means of a linear programming model with the link restoration scheme. The results have demonstrated that the utilization level of the network decreases substantially with the set up of the optimal LSP tunnels if compared with the utilization level that is observed performing only an IGP routing.

In this work we will show that, with a hybrid optimization algorithm implementing the path restoration scheme, the performances of the network management can be further improved.

\section{THE OPTIMIZATION MODEL}

The hybrid IGP/MPLS optimization problem has been formulated as a linear programming model that relies on the Multi Commodity Min Cost Flow Problem. The flow variables of our problem are of two types: one type referring to the ISIS flow variables and the other type referring to the MPLS tunnels flow. Moreover, the objective function that we want to minimize is the maximum arc utilization, since our aim is to reduce the congestion level of the network. In fact, the main goal of the model is to find an optimal set of LSP tunnels, to be used in combination with the IS-IS routing, to decrease the congestion level of the network, while at the same time guaranteeing a $100 \%$ level of survivability.

A telecommunication network can be modelled as a directed graph $G=(N, A)$ where $N(n=|N|)$ is the set of the nodes (routers) of the network and $A(m=|A|)$ is the set of directed arcs $(i, j)$. Let $K(h=|K|)$ denote a set of origin-destination pairs, or commodities; for each $h \in K$, we will denote by $s^{h}$, $t^{h}$ and $d^{h}$ respectively the origin node, the destination node and the traffic demand of the commodity $h$. Let $X(m \cdot h=$ $|X|)$ be the routing matrix, whose element $x_{i j}^{h}$ is equal to the fraction of the flow of the commodity $h$ that is routed by IS-IS/OSPF along the arc $(i, j), h \in K,(i, j) \in A$. Hence, the rows of the routing matrix correspond to the arcs, and the columns correspond to the commodities, i.e., to the pairs of origin-destination nodes. The problem under investigation consists of satisfying the demands of the commodities in $K$ in such a way to minimize the maximum arc utilization of the network. The utilization of an $\operatorname{arc}(i, j)$ is defined as the total load of $(i, j)$ over the associated capacity $c_{i j}$, where the total load of $(i, j)$ is the sum over all the demands of the amount of traffic sent over $(i, j)$.

Two different formulations can be adopted: node-arc and arc-path formulations, which are classically used for Multicommodity Flow Problems [27]. Both formulations present advantages and drawbacks. In particular, the arc-path formulation has not a compact structure since it is not possible to list all the available paths in a network and use them as variables of the model; however, thanks to the column generation procedure 


$$
\begin{aligned}
\min & u_{\max } \\
& \sum_{h \in K} i s^{h} x_{i j}^{h}+\sum_{p:(i, j) \in p} f_{p} \leq u_{\max } c_{i j} \\
& \sum_{p \in P^{h}} f_{p}=d^{h}-i s^{h} \\
& \sum_{h \in K} i s^{h} x_{i j}^{h l}+\sum_{h \in K} \sum_{p \in P^{h}: l \in p} f_{p} x_{i j}^{h l}+\sum_{p:(i, j) \in p, l \notin p} f_{p} \leq u_{\max } c_{i j} \\
& f_{p} \geq 0 \\
& i s^{h} \in\left[0, d^{h}\right]
\end{aligned}
$$

$$
\begin{array}{r}
p \in P^{h}, h \in K \\
h \in K
\end{array}
$$

[27] it can be solved efficiently. On the other hand, the node arc formulation has a compact structure but usually it is disregarded for the solution of Multicommodity Flows Problems: this is due to the fact that the number of variables is very large and therefore requiring a lot of memory. The arcpath formulation of the problem combined with the column generation procedure is then generally preferred.

To formulate the model, the following data are given:

$N$ : set of nodes;

$A$ : set of arcs;

$K$ : set of commodities;

$c_{i j}:$ arc capacity, $(i, j) \in A$, [bit/s];

$d^{h}:$ traffic demand of commodity $h, h \in K$, [bit/s];

$x_{i j}^{h}$ : fraction of the traffic of commodity $h$ carried by ISIS/OSPF along the arc $(i, j)$ with the known set of metrics, $h \in K,(i, j) \in A$.

The variables of the model are denoted as follows:

$u_{\max }:$ maximum utilization over all the arcs of the network;

$i s^{h}$ share of traffic demand $d^{h}$ of the commodity $h$ carried by IS-IS/OSPF, $h \in K$; [bit/s];

$f_{p}$ share of traffic demand $d^{h}$ of the commodity $h$ carried by LSP along $p$-th path via the MPLS-TE protocol, $h \in K$, $(i, j) \in A,[\mathrm{bit} / \mathrm{s}]$.

Let $P^{h}$ be the set of paths in $G$ from the node $s^{h}$ to the node $t^{h}$, and let $P=\cup_{h \in K} P^{h}$ the set of all relevant paths. Note that each path $p \in P$ belongs to a unique commodity, which is immediately identified by the starting and ending nodes of $p$; we will sometimes denote by $h(p)$ the commodity corresponding to $p$.

The arc-path formulation of the problem is shown from equation (1) to equation (6).

The objective function (1) measures the maximum arc utilization, which has to be minimized. The left hand side of constraints (2) specifies the total amount of traffic demand traversing the arc $(i, j)$ : it is composed by the traffic routed via the IS-IS/OSPF protocol (first summation) and the traffic carried by the LSP (second summation). For each arc $(i, j)$ this value must be smaller than or equal to $u_{\max } \cdot c_{i j}$, thus constraining also the maximum utilization level $u_{\max }$. Constraints (3) ensure that the communication demand of each commodity is entirely satisfied, considering both the traffic sent via IS-IS/OSPF and that sent via MPLS-TE. The survivability constraints are shown in (4). Here, $L$ is the set of all possible failures; it is intended to be (a subset of) the set of links of the network, i.e., undirected edges. We assume that $A$ contains two directed $\operatorname{arcs}(i, j)$ and $(j, i)$ for each link of the communication network; it is therefore immediate to define when a path $p$ "hits" a failure $l \in L$. For each failure scenario a routing matrix $X^{l}\left((m-1) \times k=\left|X^{l}\right|\right)$ is defined, whose element $x_{i j}^{h, l}$ is the fraction of flow of the commodity $h$ that is routed by IS-IS/OSPF protocol along the arc $(i, j)$, when the link $l$ fails. Each of these routing matrices $X^{l}$ can be easily determined, prior to solving the model, given the fixed set of IS-IS/OSPF metrics, by computing shortest paths over a graph obtained from $G$ by removing the link $l$. We define $x_{i j}^{l}$ as the fraction of the MPLS-TE traffic, routed along the arcs $l$ under the nominal working conditions, that in case of failure of the link $l$ is rerouted along the arc $(i, j)$, via the ISIS/OSPF protocol, according to the path restoration strategy. Note that, the rows of the routing matrices correspond to the arcs, and the columns correspond to the commodities. Hence, $x_{i j}^{l}$ is the element of the routing matrix $X^{l}$ corresponding to the arc $(i, j)$. The survivability constraints (4) are the most innovative features of the proposed model. Note that they are strictly dependent on the specific re-routing technology (path restoration). In particular, once a link fails, the entire flow along the path is re-routed like a commodity that has origin and destination in the ingress and egress nodes of the commodity. The routing is performed using pre-computed paths only dependent on the IS-IS/OSPF metrics. Constraint (5) is a non-negativity constraint. It is important to pay attention to constraint (6), which states that variable $i s^{h}$ is a continuous variable, making the model entirely linear. Note that, if $i s^{h}$ had been defined as binary, which means that every commodity $h$ is routed by only IS-IS/OSPF or by only MPLS-TE, the problem would have become a Mixed Integer Program that is known to be harder to solve with respect to Linear Programs.

We can see that, this formulation has a small number of constraints equal to $|A|+|K|+(|A|-2) \times(|A| / 2)$, but it has a number of variables that is possibly exponential in $|N|$ and $|A|$. Thus, the arc-path formulation (unless on very small instances) is not a compact formulation because the number of all possible paths may be astronomical. A well- 
known way to solve generalized Multi Commodity Min Cost Flow Problems with the arc-path formulation is the column generation approach.

\section{A. Column Generation}

The column generation procedure [27] can be summarized as the following. The procedure starts by solving a master problem built with a reduced set of variables with respect to the initial problem. Then, the values of the dual solutions are gathered in order to calculate the value of the reduced cost of the paths that are eligible to enter the problem. If the reduced cost of a path is negative, then the insertion of the column corresponding to the path into the linear program results in a better value of the objective function. This procedure is performed iteratively until no more negative reduced cost paths are found. The most important issue in performing a column generation procedure is the definition of the expression of the reduced cost that depends on the structure of the constraints matrix.

In our case, the constraints matrix is composed of three sets of constraints: the first set of constraints refers to the maximum arc capacity constraint (submatrix $B$, equation (2)); the second set is the flow conservation constraint on the nodes (submatrix $C$, equation (3)) ; the third one represents the survivability constraint (submatrix $D$, equation (4)). Therefore we will have three types of dual variables:

- $\lambda_{i j}$ are the variables associated with the arc capacity constraints (submatrix $B$ );

- $\gamma_{h}$ are the variables associated with the flow conservation constraints (submatrix $C$ );

- $\delta_{i j}^{l}$ are the variables associated with the survivability constraints (submatrix $D$ ).

We must therefore compute the shape of the generic dual constraint associated with a path $p$, i.e.,

$$
\left(\begin{array}{lll}
\lambda & \gamma & \delta
\end{array}\right)\left(\begin{array}{l}
B^{p} \\
C^{p} \\
D^{p}
\end{array}\right) \leq 0
$$

The generic element of the matrix $D_{i j, l}^{p}$ is

$$
D_{i j, l}^{p}= \begin{cases}x_{i j}^{h l} & \text { if } l \in p \\ 1 & \text { if } l \notin p,(i, j) \in p \\ 0 & \text { if } l \notin p,(i, j) \notin p\end{cases}
$$

Let $\hat{\lambda}, \hat{\gamma}$ and $\hat{\delta}$ be the dual optimal solution of the problem. Hence, the reduced cost of the path $p$ is

$$
\begin{aligned}
\bar{c}_{p}= & -\hat{\gamma}_{h(p)}-\sum_{(i, j) \in p} \hat{\lambda}_{i j}-\sum_{l \in p}\left(\sum_{(i, j) \in A} x_{i j}^{h l} \hat{\delta}_{i j, l}\right) \\
& -\sum_{(i, j) \in p} \sum_{l \notin p} \hat{\delta}_{i j, l}
\end{aligned}
$$

The column generation procedure is summarized in the following. We consider a reduced set of paths $\operatorname{RedP} \subset P$. We solve the primal problem and we get the dual optimal solution $\hat{\lambda}, \hat{\gamma}, \hat{\delta}$. Then, to understand if there is a path that, added to the set RedP can improve the solution, we must find whether there is a path $\hat{p}_{h}$ with negative reduced cost. If the answer is "no" then the current solution is optimal, otherwise $\operatorname{RedP}=\operatorname{RedP} \cup\left\{\hat{p}_{h}\right\}$ and we can iterate.

Note that, in general, column generation approaches may have issues with initialization: the initial set of paths $\operatorname{RedP}$ must be such that the primal problem $P_{R e d P}$ has a feasible solution (otherwise the dual problem $\left(\right.$ Dual $\left._{\text {RedP }}\right)$ is unbounded above, and therefore $(\hat{\lambda}, \hat{\gamma})$ is not defined). This is not necessarily trivial to obtain. In our case, this is instead very easily obtained: the restricted master problem is feasible even if $\operatorname{RedP}=\emptyset$, since the $i s^{h}$ variables alone suffice for constructing a feasible solution.

However, the shortest path problem to be solved is still complex because path costs are not an additive function of the traversed arcs. In fact, the survivability constraints add two terms in the expression of the cost of the arc.

If the failed link $l$ belongs to the path $p$, the extra term

$$
\zeta_{l}^{h}=\sum_{(i, j) \in A} x_{i j}^{h l} \hat{\delta}_{i j, l}
$$

has to be added to the cost. This cost is associated to links rather than to arcs, but nonetheless it would be easy to incorporate it into a standard shortest path procedure (it is associated to both directed arcs corresponding to the link, thus defining $\zeta_{i j}^{h}$ in the obvious way).

The real issue is the term

$$
\sum_{(i, j) \in p} \sum_{l \notin p} \hat{\delta}_{i j, l}
$$

which depends both on arcs which do belong to $p$ and on arcs which do not belong to $p$.

The separation —or pricing - problem to be solved is therefore a quadratic shortest path problem. For a given commodity $h$, it can be formulated as shown from equation (7) to equation (9). As before, $\hat{\delta}_{i j, r v}$ is equal to $\hat{\delta}_{i j, l}$ for both $\operatorname{arcs}(r, v)$ and $(v, r)$ that are the "orientations" of the undirected link $l$.

Being this problem NP-Hard, solving it with a mixed integer linear programming problem solver is only feasible for small-size instances. However, for our purposes we can try to solve it heuristically.

\section{B. Heuristic Approach}

A large set of heuristics can be constructed starting from the following simple idea. Fix a commodity $h$ and any subset $V \subseteq A$ of the arcs, and define, for each arc $(i, j)$, the modified cost

$$
\xi_{i j}^{V, h}=-\hat{\lambda}_{i j}-\sum_{(i, j) \in A} x_{i j}^{h l} \hat{\delta}_{i j, l}-\sum_{l \notin V} \hat{\delta}_{i j, l} .
$$

That is, $V$ is a "fixed estimate" of the unknown optimal path $p$. One can easily solve the shortest path problem with arc costs $\xi_{i j}^{V, h}$, thus an iterative process can be set up where:

- for a fixed $V$, the shortest path is solved with costs $\xi_{i j}^{V, h}$ yielding a path $p$; 


$$
\begin{array}{r}
\min \sum_{(i, j) \in A}\left(-\hat{\lambda}_{i j}-\zeta_{i j}^{h}-\sum_{(r v) \in A} \hat{\delta}_{i j, r v}\left(1-y_{r v}\right)\right) y_{i j} \\
\sum_{(j, i) \in B S(i)} y_{j i}-\sum_{\substack{(i, j) \in F S(i) \\
y_{i j} \in\{0,1\}}} y_{i j}=\left\{\begin{array}{ll}
-1 & \text { if } i=s^{h} \\
1 & \text { if } i=t^{h} \\
0 & \text { otherwise }
\end{array} \quad i \in N\right.
\end{array}
$$

- the "true" reduced cost of $p$ is computed; if it is negative, then a "good" column has been found: stop.

- if $p=V$ then stop, otherwise set $V=p$ and iterate.

The process is stopped when a fixed number of iterations is obtained. It can be started with $V=\emptyset, V=A$, or with any randomly generated set $V$ (in this work the best performance of the algorithm has been obtained with the initialization of the set $V$ with randomly generated sets). When the process stops (without having found a negative reduced cost path), it can be easily restarted with a randomly generated $V$. Any metaheuristic technique (simulated annealing, tabu search) can be used to improve the quality of the obtained solution.

\section{RESULTS}

Five different topologies of telecommunication networks have been used to test the proposed model. One of them is a synthetic network, two of them are real backbone networks and the other two are test network instances that are available in literature.

The first network under study is a randomly generated synthetic network composed by $n=8$ nodes and $m=36$ directed arcs, each of them having a maximum capacity of 1000 Mbps. The traffic matrix used during the test has been generated aiming at producing congestion over some links in case of single link failure.

The second instance that we analyze is an artificial network (Test network) that has been first introduced by [28]. It is composed by $n=9$ nodes and 15 bidirected edges that compose $m=30$ arcs. The experiments have been performed considering three origin-destination traffic demands.

The third instance is the Italian portion of Tiscali International Network (TINet) [29]. The network, whose topology is shown in Figure 1, is composed of 18 nodes and 54 arcs with different capacity ranged from 1 Gbps to $2.5 \mathrm{Gbps}$. The traffic matrix, composed of 306 commodities, has been built thanks to the collaboration of the ISP's network operators and managers and considers a worst case scenario.

The fourth network analyzed is the Géant network [30], the European network that connects universities and research centers, composed of 23 nodes and 38 links. The topology is shown in Figure 2. In [31] several traffic matrices for the Géant network have been provided; in particular the dataset is composed by traffic matrices generated monitoring the traffic every 15 minutes for a period of almost 4 months. In order to identify a worst case matrix, we have analyzed the link occupation levels corresponding to the traffic matrices of one

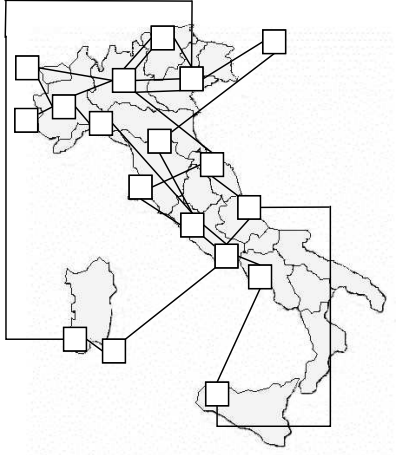

Fig. 1. Tinet Network

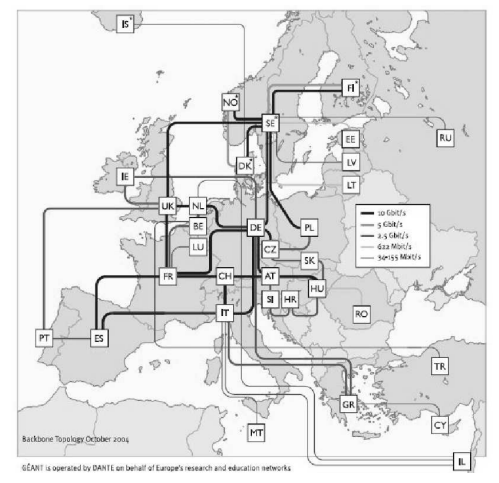

Fig. 2. Géant International Network

week and the one with the highest level of mean and maximum link occupation has been selected.

The last network under study is part of the Zuse Institute Berlin's (ZIB) SNDlib [32], which is a library of test instances for Survivable fixed telecommunication network design. The instance, related to a capacity planning problem, has been provided by the INTEC Broadband Communication Networks research group (IBCN), which is a research institute founded by the Flemish Government, focusing on information \& communication technology (ICT) in general, and applications of broadband technology in particular [33].

The network consists of $n=37$ nodes widely spread in the European territory, and $m=114$ directed arcs. The traffic matrix is given as part of the instance with $n(n-1)=1332$ commodities. During the test, the capacity of the links has been fixed and the original demands values have been scaled in order to produce congestion over some links in case of single link failure. 
TABLE I

NETWORK CHARACTERISTICS

\begin{tabular}{|c|c|c|c|c|}
\hline & $\begin{array}{c}\text { \# of } \\
\text { nodes }\end{array}$ & $\begin{array}{c}\text { \# of } \\
\text { links }\end{array}$ & $\begin{array}{c}\text { \# of } \\
\text { demands }\end{array}$ & $\begin{array}{c}\text { Avg. \# of } \\
\text { hops per demand }\end{array}$ \\
\hline Random & 8 & 36 & 56 & 1,60 \\
\hline Test & 9 & 30 & 3 & 2,25 \\
\hline Tinet & 18 & 54 & 294 & 2,98 \\
\hline Géant & 23 & 74 & 445 & 3,07 \\
\hline IBCN & 37 & 114 & 1332 & 3,72 \\
\hline
\end{tabular}

In Table I the characteristics of all the five benchmark networks are summarized. For each network instance, the number of nodes, the number of links, the number of point to point demands and the average number of hops for each demand are listed.

The results are shown in Table II. They have been obtained solving the LP model for the five instances and they have been compared with those obtained by using only the ISIS/OSPF routing. The first two columns indicate the maximum arc utilization obtained with the IS-IS/IGP routing and by solving the LP model. The last three columns give us some information related to the solution of the LP model: the total number of LSPs, the average length of the LSPs, the average stretch of the LSP tunnels with respect to the IS-IS routing and the computational time needed to solve the problem. The stretch is a metric that quantifies how long are the LSP tunnels (in terms of number of hops) with respect to the IS-IS/IGP routing paths: it is defined as the ratio of the average length of an LSP tunnel over the average length of a path with the IS-IS/IGP routing protocol.

First of all, we can see that for all the instances, the congestion levels of the networks decrease substantially by adding a small number of LSP tunnels (if compared with the total number of traffic demands). For the Tinet network, we can see that, by using only the IS-IS/IGP routing, we have a congestion scenario. This is represented by the fact that the maximum link utilization is $128 \%$, which means that the packets are dropped. The congestion scenario is avoided by adding only 8 LSPs. As far as the Geánt network is concerned, the use of only 3 LSPs reduces the congestion level of the network of more than the $20 \%$.

For all the instances the average stretch is bigger than one: this means that the LSP tunnels are on average longer than the IS-IS/IGP routing paths. As far as the computational time is concerned, we can say that it depends on the size of the network and on the maximum number of iteration we want to perform in the heuristic procedure.

TABLE II

RESULTS

\begin{tabular}{|c|c|c|c|c|c|}
\hline & $\begin{array}{c}\text { IS-IS/OSPF } \\
u_{\max } \%\end{array}$ & $\begin{array}{c}\text { LP model } \\
u_{\max } \%\end{array}$ & \# LSP & $\begin{array}{c}\text { Avg. } \\
\text { Stretch }\end{array}$ & $\begin{array}{c}\text { Time } \\
(\mathrm{sec})\end{array}$ \\
\hline Random & 70 & 35 & 10 & 1,42 & 0,72 \\
\hline Test & 60 & 40 & 4 & 1,22 & 0,88 \\
\hline Tinet & 128 & 83 & 8 & 1,29 & 9,1 \\
\hline Geánt & 97,8 & 76,1 & 3 & 1,08 & 31,72 \\
\hline Ibcn & 73,5 & 55,3 & 489 & 1,95 & 444,76 \\
\hline
\end{tabular}

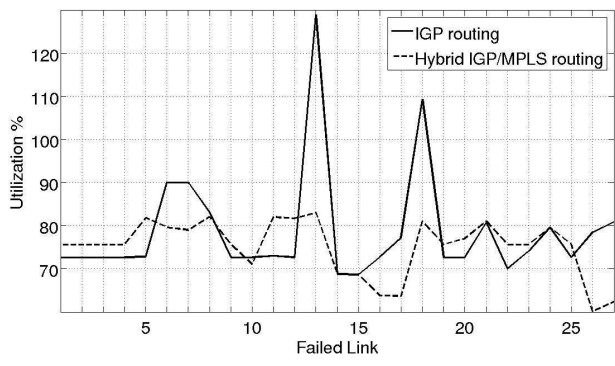

Fig. 3. Objective function for Tinet Network

In Figure 3, the comparison of the link congestion level of the Tinet Network for the IGP protocol and the hybrid IGP/MPLS routing is shown. The level of utilization of the network is depicted in function of the failed link. The spike corresponds to the most critical failure scenario. As we can see from the figure, the congestion scenario is avoided by balancing the traffic load throughout the network.

Tests have been performed in order to compare the performances of the hybrid IGP/MPLS link restoration optimization problem proposed by [3] with our results. The results are shown in Table III. We can see that with the LP model that we present in this paper the link congestion level is lower for some of the networks ( the Test and the IBCN networks) and the number of LSP tunnels to be set up is always limited. These results are due to the fact that the path restoration is more efficient than the link restoration technique.

As previously mentioned, the link restoration re-routing technique may suffer of backhauling, that is the fact that a link is used more than once along a route towards a destination. This fact is clear if we observe Figure 4. The scenario of a single commodity from node 1 to node 9 is shown for the Test network considering the link restoration and the path restoration re-routing schemes after the failure of the link $1-2$ : in bold the primary LSP tunnel are indicated, with the dashed line the IS-IS backup paths. We can see from Figure 4(a) how the re-routing of the traffic results in the utilization of the link $2-3$ in both directions. On the other hand, in Figure 4(b) it is shown how the backhauling effect is completely avoided with the path restoration re-routing scheme.

\section{Conclusions}

In this paper, a linear programming model has been presented, that jointly use the IGP routing protocols and the MPLS-TE technology to minimize the maximum link utiliza-

TABLE III

COMPARISON RESTORATION SCHEMES

\begin{tabular}{|c|c|c|c|c|}
\hline \multirow{2}{*}{} & \multicolumn{2}{|c|}{ Present Paper } & \multicolumn{2}{c|}{ Work [3] } \\
\cline { 2 - 5 } & $u \max \%$ & \# LSP & umax \% & \# LSP \\
\hline Random & 35 & 10 & 35 & 53 \\
\hline Test & 40 & 4 & 42 & 6 \\
\hline Tinet & 83 & 8 & 83 & 80 \\
\hline Géant & 76,1 & 3 & 76,1 & 210 \\
\hline Ibcn & 55,3 & 489 & 65,49 & 585 \\
\hline
\end{tabular}




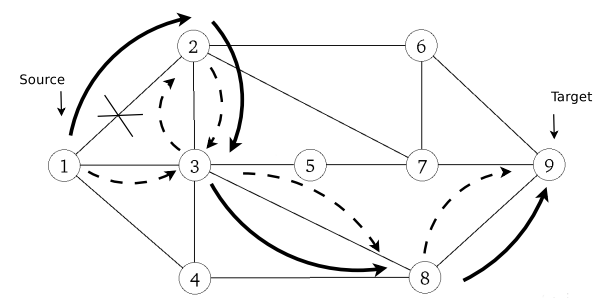

(a) Link Restoration Re-Routing

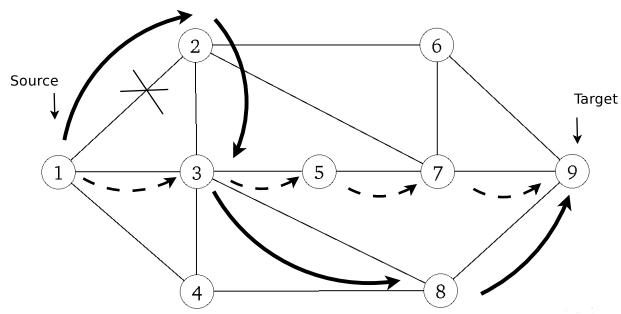

(b) Path Restoration Re-Routing

Fig. 4. Re-Routing Comparison for Test Network

tion in backbone networks. The proposed approach embeds in a unique overall model the two different technologies providing $100 \%$ survivability in single link failure scenarios. Survivability of the service delivery is guaranteed following Path Restoration scheme. The model solution is obtained in nearly real time even for real telecommunication networks. Furthermore, it is possible to implement the results of the model with a very limited intervention of the network operators. Our results show that by constructing a few LSPs we could improve network efficiency by reducing the maximum link utilization, while simultaneously keeping full survivability.

\section{ACKNOWLEDGEMENT}

This work, has been partially supported by Tiscali S.p.A within the framework of the ICE Lab (Information and Communications Engineering Academy Joint Laboratory). The authors would like to thank Paolo Susnik and Giorgio Lembo for the useful discussions.

\section{REFERENCES}

[1] J.K. Lenstra and A.H.G. Rinnooy-Kan. On general routing problems. Networks, 6:273-280, 1976.

[2] B. Fortz and M. Thorup. Increasing internet capacity using local search. Computational Optimization and Applications, 29(1):13-48, 2004.

[3] D. Cherubuni, A. Fanni, A. Frangioni, C. Murgia, M.G. Scutellá, and P. Zuddas. A linear programming model for traffic engineering in $100 \%$ survivable networks under combined is-is/ospf and mpls-te protocols. Technical report, 2008. http://compass2.di.unipi.it/TR/Files/TR08-24.pdf.gz.

[4] E.W. Dijkstra. A note on two problem in connexion with graphs. Numerische Mathematik, 1:269-271, 1959.

[5] Requirements for traffic engineering over mpls, rfc 2702.

[6] Requirements for traffic engineering over mpls, rfc 4090
[7] G. Iannaccone, C. Chuah, R. Mortier, S. Bhattacharyya, and C. Diot. Analysis of link failures in an IP backbone. In Proc. of ACM SIGCOMM Internet Measurement Workshop 2002, Marseille, France, Nov 2002, Nov 2002.

[8] A. Markopoulou, G. Iannaccone, S. Bhattacharyya, C. Chuah, and C. Diot. Characterization of failures in an IP backbone. In IEEE Infocom 2004, IRC-TR-04-015, Hong Kong, China, March 2004, 2004.

[9] H. Luss and R.T. Wong. Survivable telecommunications network design under different types of failures. Systems, Man and Cybernetics, Part A, IEEE Transactions on, 34(4):521-530, July 2004.

[10] B. Fortz and M. Thorup. Internet traffic engineering by optimizing OSPF weights. 2000.

[11] B. Fortz and M. Thorup. Robust optimization of OSPF/IS-IS weights. INOC 2003, 2003.

[12] A. Fanni D. Cherubini and C. Murgia. Is-is/ospf weights optimization for survivable backbone networks: a tabu search metaheuristic approach. 2006.

[13] M. Ericsson, M. Resende, and P. Pardalos. A genetic algorithm for the weight setting problem in OSPF routing. Combinatorial Optimization, 6:299-333, 2002.

[14] E. Mulyana and U. Killat. A Hybrid Genetic Algorithm Approach for OSPF Weight Setting Problem. In Peroc. of the $2^{\text {nd }}$ Polish-German Teletraffic Symposium PGTS, 2004.

[15] L. S. Buriol, M. G. C. Resende, C. C. Ribeiro, and M. Thorup. A memetic algorithm for OSPF routing. In Proc. of the $6^{\text {th }}$ INFORMS Telecom, pages 187-188, 2002.

[16] J. Harmatos. A heuristic algorithm for solving the static weight assignment optimisation problem in OSPF networks. In Proc. of of Global Internet Conference, 2002.

[17] S. Beker, N. Puech, and V. Friderikos. A Tabu Search heuristic for the offline MPLS reduced complexity layout design problem. In Networking, pages 514-525, 2004.

[18] S. Cerav Erbas and C. Erbas. A multiobjective off-line routing model for MPLS networks. In Proc. of the 18th International Teletraffic Congress, 2003.

[19] A. Elwalid, C. Jin, S. H. Low, and I. Widjaja. MATE: MPLS adaptive traffic engineering. In INFOCOM, pages 1300-1309, 2001.

[20] A. Elwalid, C. Jin, S. Low, and I. Widjaja. Multi-path adaptive traffic engineering. Computer Networks, 40:695-709, 2002.

[21] Anton Riedl. Optimized routing adaptation in IP networks utilizing OSPF and MPLS. In IEEE 2003 International Conference on Communications (ICC), Anchorage, USA, May 2003, May 2003.

[22] E. Mulyana and U. Killat. Optimization of ip networks in various hybrid igp/mpls routing schemes. In Proceedings of the 3rd Polish-German Teletraffic Symposium PGTS 2004, Dresden Germany, sep 2004.

[23] F. Skivée, S. Balon, and G. Leduc. A scalable heuristic for hybrid IP/MPLS traffic engineering - case study on an operational network. In Proc. of IEEE International Conference on Networks (ICON). IEEE Press, September 2006.

[24] D. Yuan. A bi-criteria optimization approach for robust OSPF routing. In $6^{\text {th }}$ IEEE Workshop on IP Operation Management (IPOM), 2003.

[25] L. Li, M. Buddhikot, C. Chekuri, and K. Guo. Routing Bandwidth Guaranteed Paths with Local Restoration in Label Switched Networks. IEEE Journal on Selected Areas in Communications, 23(2), 2005.

[26] Yu Liu and D. Tipper. Spare capacity allocation for non-linear cost and failure-dependent path restoration. In Proceeding of the $3^{\text {rd }}$ International Workshop on Design of Reliable Communication Networks, DRCN, 2001.

[27] R. K. Ahuja, T. L. Magnanti, and J. B. Orlin. Network flows: theory, algorithms, and applications. Prentice-Hall, Inc., Upper Saddle River, NJ, USA, 1993.

[28] M. Patel, R. Chandrasekaran, and S. Venkatesan. A comparative study of restoration schemes and spare capacity assignments in mesh networks. Computer Communications and Networks, 2003. ICCCN 2003. Proceedings. The 12th International Conference on, pages 399404, Oct. 2003.

[29] Tiscali International Network.

[30] http://www.geant.net/.

[31] S. Uhlig, B. Quoitin, J. Lepropre, and S. Balon. Providing public intradomain traffic matrices to the research community. ACM SIGCOMM Computer Communication Review, 36(1):83-86, Jan. 2006.

[32] http://sndlib.zib.de/home.action.

[33] www.ibcn.intec.ugent.be. 